\title{
LOS LÍMITES DEL PARADIGMA ESTRATÉGICO ISRAELÍ
}

\author{
Guillem Colom ${ }^{1}$ \\ Universidad Pablo de Olavide \\ Instituto General Gutiérrez Mellado (UNED)
}

\begin{abstract}
Resumen:
Este artículo repasa la evolución del paradigma estratégico israelí desde la creación del Estado de Israel en 1948 hasta la guerra de verano de 2006, un conflicto cuyo desenlace no sólo puso de manifiesto la erosión del modelo de ciudadano-soldado vigente hasta la fecha, la inadecuación de los tradicionales planteamientos estratégicos al nuevo entorno regional, o las carencias operativas de unas fuerzas armadas orientadas al mantenimiento de la supremacía militar convencional hebrea en Oriente Medio; sino también reveló el nuevo rostro de la guerra. Todos estos elementos están comportando una redefinición de la política de seguridad y defensa israelí para adaptarla a la nueva situación estratégica de la región.
\end{abstract}

Palabras clave: Israel, Oriente Medio, estrategia, política de seguridad, política de defensa, fuerzas armadas, asuntos militares.

\begin{abstract}
:
The article analyzes the evolution of Israel's strategic thought since its conception in 1948 until the 2006 IsraeliHezbollah war, a conflict which not only revealed the erosion of Israel's model of citizen-soldier, the inadequacies of its traditional strategic paradigm to the new regional environment and the operational limitations of the Israel's Defense Forces (IDF) when countering asymmetric and hybrid threats; but also exposed the changing face of war. Those factors are compelling Israel to redefine its security and defence policies as a means to adapt the country to the changing strategic landscape
\end{abstract}

Keywords: Israel, Middle East, strategy, defense policy, military policy, armed forces, military affairs.

Copyright (C) UNISCI, 2011.

Las opiniones expresadas en estos artículos son propias de sus autores, y no reflejan necesariamente la opinión de UNISCI. The views expressed in these articles are those of the authors, and do not necessarily reflect the views of UNISCI.

\footnotetext{
${ }^{1}$ Guillem Colom Piella es Doctor en Paz y Seguridad Internacional (IUGGM-UNED), Master y doctorando en Relaciones Internacionales (UAB), Licenciado en Sociología (UAB) y en Ciencias Politicas (UAB). Profesor del área de Ciencia Política de la Universidad Pablo de Olavide y del Instituto General Gutiérrez Mellado (UNED). Líneas de investigación: seguridad y defensa, relaciones transatlánticas, asuntos militares, estudios estratégicos. Email: guillemcolom@hotmail.com, gcolpie@upo.es.
} 
"The Egyptians could run to Egypt, the Syrians into Syria. The only place we could run was into the sea, and before we did that we might as well fight"

Golda Meir, Primera Ministra de Israel (1969-1974)

\section{Introducción}

La guerra que se produjo en verano de 2006 entre Israel y Hezbollah representa un punto de inflexión en el paradigma militar hebreo. La Operación Recompensa Justa, nombre que recibe la campaña militar israelí iniciada en Julio de 2006 con objeto de liberar a los soldados hebreos capturados por las milicias de Hezbollah, terminar con los ataques de cohetes a las ciudades israelíes e implementar la Resolución 1559 (2004) del Consejo de Seguridad de las Naciones Unidas para desarmar y desmantelar las milicias chiítas del sur del Líbano, arrancó como una operación limitada pero escaló hacia una guerra abierta en la que Israel sufrió un duro revés a manos de Hezbollah: la sucesión de errores políticos y militares precipitaron una campaña improvisada y costosa que no logró conquistar los objetivos militares y puso en entredicho la supuesta invencibilidad de las todopoderosas Fuerzas de Defensa Israelíes (FDI).

Las causas de esta derrota son muchas y muy diversas (éstas comprenden desde una incorrecta planificación militar y un lento proceso de toma de decisiones a la erosión del arquetipo de ciudadano-soldado, los perniciosos efectos de la americanización de las FDI o la inadecuación del modelo de seguridad nacional vigente hasta la fecha); pero todas ellas están relacionadas con la erosión del tradicional paradigma estratégico que Israel ha ido articulando a lo largo de los años.

Es por ello que el presente trabajo analizará los antecedentes y evolución de este modelo estratégico que tanta efectividad ha demostrado durante las décadas pasadas para garantizar la seguridad nacional del Estado de Israel, y que los enormes cambios acaecidos en el entorno estratégico regional y en la sociedad hebrea están obligando a redefinir.

\section{El paradigma estratégico israelí}

El sueño de Theodor Herzl de crear una nación judía en Palestina se hizo realidad en el año 1948, cuando el sionismo, el holocausto nazi y la descolonización de Oriente Próximo facilitaron la obtención de un amplio acuerdo entre la Comunidad Internacional para fundar el Estado de Israel. No obstante, sus vecinos árabes - contrarios a los asentamientos judíos desde tiempos inmemoriales - emprendieron un conflicto que se ha prolongado durante más de sesenta años y en el que Israel ha sobrevivido gracias a su potencial militar, el más poderoso de Oriente Medio, y a su total determinación por emplearlo para hacer frente a cualquier amenaza.

Y es que a lo largo de sus seis décadas de existencia, Israel nunca ha articulado oficialmente una Estrategia de Seguridad Nacional que identifique los grandes objetivos del país (ends), exponga las distintas maneras de conseguirlos (ways) y perfile los medios a emplear para alcanzarlos (means). Solamente ha manifestado su plena determinación por defender la integridad del Estado con todos los medios posibles, una vaguedad que hasta la fecha le ha permitido responder con gran dinamismo a cualquier cambio en la región, pero 
siempre garantizando que cualquier violación del status quo dictado por la pequeña nación hebrea tendría, inexorablemente, una respuesta inmediata y resolutiva ${ }^{2}$.

Tal indefinición ha permitido que Israel haya utilizado su vasto potencial militar de maneras muy distintas a lo largo de su historia ${ }^{3}$; aunque siempre fundamentándose más en el pragmatismo y en el oportunismo que en análisis razonados o estrategias previamente trazadas y delimitadas ${ }^{4}$. Este pragmatismo político-militar, fundamentado en una doctrina de seguridad que responde a fines políticos pero sin explicitar los medios militares y dotando a sus fuerzas armadas de una enorme autonomía estratégica ${ }^{5}$, impidió hasta el año 1999 la creación de un Consejo de Seguridad Nacional que analizara la situación geopolítica de Israel y planteara una estrategia coherente a largo plazo que articulara y armonizara sus necesidades políticas con sus capacidades militares ${ }^{6}$. No obstante, desde su fundación este órgano asesor del Primer Ministro y del Gobierno israelíes nunca ha tenido una función clara ni destacada al estar sometido a presiones provocadas por conflictos de intereses - el Ministerio de Defensa, Asuntos Exteriores y las Fuerzas Armadas no están dispuestas a ver reducido sus influencias políticas en asuntos de seguridad nacional - y por la reticencia de la plana mayor de las FDI, contrarias a reconocer la necesidad de un órgano civil que fiscalice las decisiones estratégicomilitares y limite la tradicional autonomía de las Fuerzas Armadas del país. Sin embargo, ante el desastre cosechado en la Operación Recompensa Justa (2006) y las recomendaciones planteadas por la Comisión Winograd - que evaluó la actuación del Gobierno y las Fuerzas Armadas hebreas en esta campaña - es previsible que el Consejo de Seguridad Nacional adquiera la relevancia y autoridad necesarias para guiar la política de seguridad y defensa hebrea en este nuevo entorno estratégico dominado por la complejidad y la incertidumbre ${ }^{7}$.

Esta particular y ambigua situación que ha mantenido Israel desde su fundación hasta nuestros días ha sido juzgada de manera desigual por la comunidad de defensa israelí. Hasta fechas recientes se tendía a sostener que este pragmatismo respondía a una situación concreta y la inexistencia de una Estrategia de Seguridad Nacional nunca había amenazado la

\footnotetext{
${ }^{2}$ De hecho, a pesar de las demostraciones de fuerza y las acciones ofensivas que Israel ha realizado a lo largo de su historia para disuadir a sus enemigos o desactivar cualquier amenaza, los distintos gobiernos del país sólo han expuesto su voluntad de evitar cualquier conflicto por medios políticos y mediante una disuasión creíble. En este sentido, véase el clásico trabajo de Ben-Horin, Yoav y Posen, Barry (1981): Israel's Strategic Doctrine, Santa Monica, RAND Corporation; realizado a petición del Pentágono y actualmente desclasificado y disponible en la red, en: www.rand.org/pubs/reports/2007/R2845.pdf; o las obras de Handel, Michael: "The Evolution of Israeli Strategy: the Psicology of Insecurity and the Quest for Absolute Security”, en Murray, Williamson; Knox, Macgregor y Bernstein, Alvin (eds.) (1994): The Making of Strategy: Rulers, States, and War, Nueva York, Cambridge University Press, pp. 534-578; o Tal, Israel (2000): National Security: The Israeli Experience, Westport, Praeger Publishers.

${ }^{3}$ Cohen, Stuart e Inbar, Ephraim: “A Taxonomy of Israel's Use of Force”, Comparative Strategy Vol. 10 № 2 (Abril 1991), pp. 9-15, en: www.informaworld.com/index/782379359.pdf

${ }^{4}$ Inbar, Ephraim (1991): Rabin and Israel's National Security, Baltimore, John's Hopkins University Press

${ }^{5}$ Heller, Mark (2000): Continuity and Change in Israeli Security Policy, Adelphi Paper ${ }^{\circ}$ 335, Londres, International Institute for Strategic Studies

${ }^{6}$ Aunque la Comisión Agranat - instaurada después de la Guerra del Yom Kippur con objeto de depurar responsabilidades y subsanar los errores cometidos en este conflicto - propuso la creación de un órgano similar al Consejo Nacional de Seguridad estadounidense, la oposición política y militar hicieron que esta iniciativa se mantuviera congelada hasta finales de siglo, cuando el Primer Ministro Netanyahu logró su definitivo establecimiento en virtud de la Resolución Gubernamental 4889, del 7 de Marzo de 1999.

${ }^{7}$ El sumario en inglés del Informe Final de la Comisión Winograd, presentado al Primer Ministro y al Ministro de Defensa israelíes el día 30 de Enero de 2008, puede hallarse en: www.cfr.org/publication /15385/winograd_commission_final_report.html
} 
integridad israelí ni tampoco había reducido la hegemonía del país en Oriente Medio ${ }^{8}$. Además, la ambigüedad de la doctrina nuclear hebrea había permitido al país adaptarse con fluidez a la cambiante coyuntura regional sin que ello menoscabara su poder disuasorio ${ }^{9}$. No obstante, son cada vez más los expertos que afirman que los nuevos retos y amenazas que se ciernen sobre el país - que comprenden desde la expansión de las amenazas irregulares a la proliferación nuclear iraní - exigen que Israel deje de lado su tradicional ambigüedad estratégica y perfile una Estrategia de Seguridad Nacional que explicite los fines (ends), maneras (ways) y medios (means) del país; establezca las relaciones, potestades y responsabilidades de los estamentos político y militar; y formalice una doctrina nuclear explícita susceptible de garantizar la disuasión israelí ante cualquier eventualidad presente y futura $^{10}$.

\section{Condicionantes objetivos}

Una vez expuestos los pilares del paradigma estratégico israelí, un modelo basado en el pragmatismo, en la autonomía militar y las soluciones ad-hoc; seguidamente se mostrarán los condicionantes objetivos - geografía y demografía - que han marcado su configuración desde la creación del Estado en 1948.

Por un lado, la particular geografía israelí ha condicionado su doctrina estratégica, ya que su superficie originaria no sólo carece de obstáculos naturales sino que sus reducidas dimensiones le hacen extremamente vulnerable a cualquier ataque terrestre, naval o aéreo. Históricamente, esta situación ha condicionado el comportamiento de los estrategas israelíes, que afirmaron que la inexistente profundidad estratégica impedía al país sostener una guerra total en su territorio ni tampoco resistir un conflicto continuado de baja intensidad. De esta manera surgió la creencia, posteriormente codificada en doctrina, que cualquier guerra en la que Israel se viese implicado debería transferirse al territorio enemigo; por lo que el concepto de transferencia del conflicto ha tenido enormes implicaciones en la doctrina operacional de las FDI, que desde su constitución optaron por tácticas ofensivas que compensaran la menor potencia de fuego de sus ejércitos con una mayor movilidad y coordinación táctica.

Sin embargo, el territorio original de Israel, basado en la Línea Verde de 1949, cambió drásticamente como consecuencia de la guerra de los Seis Días, cuando en junio de 1967 Israel capturó numerosos territorios que le proporcionaron una vasta profundidad estratégica en el frente sur ante Egipto - el desierto del Sinaí - un obstáculo natural en el frente norte contra Siria - los Altos del Golán - y Cisjordania, que además de aportar profundidad, también protegía Israel de Jordania, formalmente en estado de guerra hasta 1994, y de Iraq, que representó una amenaza estratégica real para Israel hasta $1991^{11}$. No obstante, aunque esta

\footnotetext{
${ }^{8}$ Rodman, David: "Israel's National Security Doctrine, an Introductory Overview”, Middle East Review of International Affairs Journal, Vol. $5 \mathrm{~N}^{\mathrm{o}} 3$ (Septiembre 2001), pp. 7-13, en: www.meria.idc.ac.il /journal/2001/issue3/rodman.pdf

${ }^{9}$ Steinberg, Gerald: "The Future of Nuclear Weapons: Israeli Perspectives" ponencia presentada en el seminario Security Questions at the End of the Twentieth Century (Ginebra, 21 de Noviembre de 1996), en: www.faculty.biu.ac.il/ steing/arms/amaldi.htm.

10 Shabtai, Shay: "Israel's National Security Concept: New Basic Terms in the Military-Security Sphere", Strategic Assessment, Vol. $13 \mathrm{~N}^{\mathrm{o}} 2$ (Agosto 2010), pp. 7-18, en: www.inss.org.il/upload/(FILE) 1283416564.pdf

11 El hipotético frente oriental fue una consecuencia indirecta de la situación posterior a 1973, cuando la creciente desigualdad militar entre Israel y sus potenciales enemigos junto con el proceso de paz con Egipto, que acabó con el frente sur, comportaron que Iraq iniciara una política más ofensiva frente a Israel. Sin
} 
nueva situación territorial tuvo un limitado impacto en los principios tácticos y operacionales de las FDI, basados en la defensa a escala estratégica, sí influyó en su doctrina de seguridad puesto que estos territorios ofrecían ventajas evidentes frente a Egipto, Siria y Jordania.

El efecto más importante de esta nueva situación fue el aislamiento de Israel en caso de conflicto convencional a gran escala. Sin embargo, el control de estas áreas sentó las bases de la guerra del Yom Kippur (1973) y fue el detonante del conflicto de baja intensidad respuesta irregular frente a la supremacía israelí en el terreno convencional - en los territorios palestinos y en el sur del Líbano.

Actualmente, el pensamiento estratégico israelí pone un limitado énfasis en el control del territorio como pilar fundamental de su seguridad dada la gran capacidad que poseen sus fuerzas armadas para proyectar su poder y la proliferación de armamento no-convencional y de vectores capaces de transportarlo. Esta situación hace suponer que, en caso de desatarse una conflagración convencional, el principal objetivo de las FDI sería la destrucción del poder militar árabe y su infraestructura político-económica, renunciando a la conquista territorial como objetivo militar ${ }^{12}$.

Por otro lado, la vasta desigualdad demográfica existente entre Israel y sus vecinos árabes definió una postura militar que ha ido evolucionando con el paso del tiempo. Sin embargo, la inmigración judía, el crecimiento natural de la población y el incremento del diferencial militar entre Israel y sus adversarios han convertido en irrelevante tal desequilibrio, por lo que la demografía ha dejado de ser un condicionante de la seguridad hebrea.

Desde un punto de vista estratégico, tal desproporción demográfica entre Israel y sus adversarios permitía a los árabes mantener ejércitos profesionales mientras que Israel no. En consecuencia, para superar esta situación se consideró vital disponer de unas FDI estructuradas a modo de milicia, con un núcleo profesional encargado de preservar la seguridad diaria del país y prepararse para una guerra convencional a gran escala; mientras que en caso de guerra, el ejército multiplicaría su tamaño gracias a la llamada a filas en masa de hombres y mujeres. Mientras en tiempo de paz esta estructura maximizaba las capacidades demográficas israelíes puesto que garantizaba la estabilidad socioeconómica del estado, en tiempo de guerra - cuando la población se movilizaba en masa - las operaciones militares debían ser resueltas rápida y decisivamente para evitar la parálisis de la economía israelí.

Este modelo de fuerzas armadas se mostró muy eficaz durante las guerras de 1956 y 1967, cuando Israel gozaba de la iniciativa estratégica y conducía operaciones ofensivas. Sin embargo, durante la guerra del Yom Kippur, cuando las fuerzas egipcias y sirias atacaron a

embargo, la guerra entre Irán e Iraq (1980-88) limitó enormemente esta amenaza que acabó por desaparecer después de la Guerra del Golfo de 1991.

${ }^{12}$ No obstante, entre los israelíes más ortodoxos se mantiene la tendencia en plantear la seguridad del país en términos territoriales y, por lo tanto, de profundidad estratégica. El argumento más elaborado procede del profesor Gerald Steinberg, que entiende que la proliferación de armamento de destrucción masiva en Oriente Medio comportará inexorablemente la aparición de un entorno de disuasión multipolar que socavará el monopolio disuasorio hebreo y quebrantará la superioridad estratégica israelí, estadounidense y aliada en el Mediterráneo. En el caso que se diera este improbable equilibrio estratégico en la región, aumentaría la probabilidad de escalada bélica convencional, por lo que sería preciso volver a enfatizar la importancia del control del territorio como medio indispensable para la defensa de Israel. Steinberg, Gerald: "Israel looks over the horizon: responding to the threats of weapons proliferation", Jerusalem Center for Public Affairs, No 457 (Julio de 2001), en: www.jcpa.org/j1/vp457.htm; o "Rediscovering Deterrence after September 11, 2001", Jerusalem Center for Public Affairs, № 467 (Diciembre 2001), en: www.jcpa.org/j1/vp467.htm 
Israel por sorpresa, las FDI estuvieron al borde del colapso ya que sus fuerzas permanentes fueron sobrepasadas por los ejércitos atacantes, obligando al Tzáhal a luchar defensivamente reforzando las líneas de frente y movilizando completamente a la reserva. Aún así, la contraofensiva israelí infligió un gran número de bajas entre los atacantes, forzando a las superpotencias a imponer un alto el fuego que salvó a Siria y Egipto de una derrota decisiva.

Como consecuencia de la guerra de 1973 - y una vez la Comisión Agranat depurara las responsabilidades de las fuerzas armadas y realizara varias recomendaciones con objeto de mejorar la capacidad de alerta temprana, el análisis de inteligencia y la capacidad de respuesta de las FDI - el Gobierno y el ejército israelíes articularon su doctrina nuclear y sus aplicaciones en la esfera político-militar. Asimismo, las fuerzas convencionales incrementaron su tamaño, ampliaron su catálogo de capacidades y redefinieron su organización interna con objeto de lograr una superioridad tecnológica que sirviera de multiplicador en el campo de batalla y permitiera al país mantener la estructura miliciana vigente hasta entonces.

No obstante, a partir de la década de los noventa, factores como la presión social (una menor voluntad de la población por formar parte de las fuerzas armadas), la evolución demográfica (el incremento natural de la población y la inmigración judía de terceros países) y las nuevas tecnologías, han permitido incrementar notablemente el volumen de fuerza de las FDI mientras se han reducido las levas y se ha profesionalizado gradualmente parte de la fuerza $^{13}$. No obstante, esta profesionalización no ha comportado en absoluto la desaparición del ejército de base miliciana porque su existencia se entiende necesaria por razones socializadoras y porque en caso de una guerra convencional a gran escala - una hipótesis cada vez más improbable - continuaría siendo esencial ${ }^{14}$.

\section{Las respuestas israelíes}

Para hacer frente a los condicionantes geográficos y demográficos, los estrategas israelíes delinearon una serie de respuestas que, con los años, han contribuido a configurar un pensamiento estratégico tácito que nunca ha sido expuesto públicamente en ningún documento oficial. Aunque este trabajo no pretende estudiar de forma exhaustiva dichas respuestas - la mayoría de las cuales se limitan al nivel táctico y se vinculan con el empleo de la fuerza - sí que se expondrán las soluciones más conocidas que el ejército y el gobierno hebreos escogieron para superar la particular situación de Israel.

\subsection{Disuasión: concepto central en la estrategia de seguridad israelí}

Israel ha sido, durante mucho tiempo, un Estado no reconocido por sus vecinos y todavía es considerado ilegítimo por parte de la Comunidad Internacional y de la opinión publica mundial. Esta particular situación le ha obligado a defender su integridad de dos formas: de manera pacífica, disuadiendo a sus oponentes de iniciar un conflicto; o por medios militares, mediante acciones preventivas con objeto de demostrar su poder y anticiparse a cualquier acción enemiga. A continuación, se examinarán las relaciones entre Israel y sus adversarios

\footnotetext{
${ }^{13}$ Cohen, Stuart A.: "Small States and Their Armies: Restructuring the Militia Framework of the Israel Defense Force", The Journal of Strategic Studies, Vol. $18 \mathrm{~N}^{\mathrm{o}} 4$ (Diciembre de 1995), pp. 78-93; en: www.informaworld.com/index/789939916.pdf

${ }^{14}$ Creveld, Martin V. (1998): The Sword and the Olive: A Critical History of the Israeli Defense Forces, Nueva York, Public Affairs
} 
tanto en tiempo de paz como en situación de guerra, cuando el país emplea su poder militar para lograr sus objetivos políticos de seguridad y garantizar el mantenimiento de su statu-quo.

La disuasión - bien sea convencional o no-convencional - es uno de los medios que tradicionalmente ha empleado el Estado de Israel para prevenir cualquier ataque; por lo que siempre ha intentado proyectar una imagen de fuerza (y la determinación por emplearla) mucho mayor que la de sus oponentes. En este sentido, el profesor David Rodman establece una división entre disuasión convencional general y específica o por negación y castigo ${ }^{15}$, actitudes siempre basadas en la demostración de una capacidad militar mucho mayor que la de sus adversarios. Contrastando con la disuasión convencional, Israel mantiene una postura mucho menos clara frente a las amenazas no-convencionales, aunque siempre ha alertado de que cualquier acción puede tener represalias masivas. En este sentido, para intentar disuadir los actos terroristas como forma de guerra asimétrica, Israel siempre ha respondido de manera desproporcionada; y para evitar el empleo de armas de destrucción masiva, Tel Aviv asegura que responderá con armamento nuclear.

Esta afirmación - la amenaza de responder con armas atómicas en caso de ataque nuclear, biológico, químico o radiológico (NBQR) - cuestiona la repetida declaración que Israel no será el primer Estado que introducirá armas no-convencionales en la región. Esta contradicción, fundamentada en la posesión real de armamento de destrucción masiva a la vez que niega su existencia, ha permitido a Israel disfrutar de los beneficios de ser una nación del selecto club nuclear en términos de disuasión y de capacidad de respuesta, pero sin sufrir las repercusiones internacionales derivadas del conocimiento de su arsenal. Además, mediante la llamada Doctrina Begin de ataques preventivos, Israel ha impedido que sus potenciales adversarios puedan desarrollar ingenios nucleares susceptibles de alterar el monopolio israelí, un hecho que se evidenció con el ataque a la planta nuclear iraquí de Osirak en 1981 y que no puede descartarse sea empleada en un futuro no demasiado lejano contra Irán.

Igualmente, es interesante apuntar que desde el año 1973 - fecha en que se elaboró la doctrina nuclear israelí - este país contempla el empleo real de armas nucleares a todos los niveles. En otras palabras, mientras a escala táctica podría usarlas para diezmar cualquier ejército invasor, a escala estratégica podría utilizar sus vectores - aviones y mísiles de largo alcance basados en tierra y de crucero en submarinos - para atacar los centros de gravedad enemigos ${ }^{16}$. Además, en última instancia Israel también podría llevar a cabo la denominada Opción Sansón: un ataque nuclear total contra sus adversarios en caso que la integridad del país estuviera realmente en peligro ${ }^{17}$.

\footnotetext{
${ }^{15}$ Rodman, Israel's National Security Doctrine, op. cit., pp. 9-11 sostiene que la disuasión general se basa en el hecho que mientras los estados árabes pueden iniciar una guerra, Israel acabará determinando su alcance e intensidad; mientras que la específica se ha construido en torno al concepto de líneas rojas explícitas que, en caso de ser traspasadas, supondrán una respuesta militar. La disuasión por negación puede definirse como la capacidad de evitar que un oponente inicie una acción porque ésta será fácilmente repelida; mientras que la disuasión por castigo entraña la capacidad de evitar que cualquier enemigo ataque Israel porqué no sólo podrá defenderse, si no también atacarlo en represalia e imponerle unos costes inaceptables.

${ }^{16}$ Los elementos de su disuasión estratégica se componen de aviones de combate capaces de transportar armamento convencional o nuclear; misiles balísticos de corto y medio alcance que proyectan el poder israelí más allá de su espacio estratégico y misiles de crucero lanzables desde submarinos, base de la respuesta y represalia convencional o nuclear del país.

${ }^{17}$ La Opción Sansón, formulada en 1966 bajo el Gobierno de Levi Eshkol, constituye la primera articulación teórica sobre el empleo de armas nucleares. Esta opción se llevaría a cabo si se daba alguno de los siguientes supuestos: si los ejércitos árabes penetraban dentro las fronteras de 1949 y amenazaban los centros de población; si las Fuerzas Aéreas Israelíes eran destruidas; si las ciudades israelíes se exponían a ataques aéreos masivos con armamento convencional, químico o biológico, o si se empleaban armas nucleares contra el
} 
A modo de conclusión cabe comentar que, tal y como sostienen Ephraim Inbar y Shmuel Sandler ${ }^{18}$, la capacidad hebrea para disuadir a sus enemigos se ha mantenido estable a lo largo del tiempo, aunque el advenimiento del conflicto de baja intensidad muestra que ésta - en forma de represalias - no ha logrado los efectos deseados sobre los grupos que emplean medios y procedimientos irregulares o híbridos, que a día de hoy constituyen una de las mayores amenazas para la seguridad diaria del país ${ }^{19}$. Igualmente, Rodman asume improbable el inicio de una guerra convencional en la región porque la cada vez mayor asimetría militar entre Israel y sus adversarios disuade a cualquier ejército árabe de atacar a Israel; y añade que es posible que se mantenga la misma disuasión nuclear a pesar de que otros países de la región hayan adquirido armamento químico y biológico u obtengan en un futuro armamento nuclear. No obstante, parece evidente que esta nueva situación, que todavía no puede considerarse de disuasión multipolar, influirá - junto con factores políticos y diplomáticos para que el nuevo modelo estratégico israelí excluya las acciones militares preventivas contra objetivos estratégicos enemigos como medio para lograr la seguridad de Israel $^{20}$.

\subsection{La predilección por guerras cortas y ofensivas}

Desde un prisma estrictamente militar, la única estrategia viable para hacer frente a los condicionantes territoriales y demográficos del país era desarrollar un tipo de acciones militares que maximizaran las capacidades israelíes - adiestramiento, movilidad táctica y coordinación de fuegos - a escala operacional y táctica, minimizando el riesgo de verse involucrados en guerras de desgaste o defensivas en las que los ratios de fuerzas son determinantes.

La solución que eligieron los estrategas israelíes fue primar las guerras cortas con la finalidad de limitar el impacto económico, los costes humanos y evitar la posibilidad de cualquier intervención diplomática o militar extranjera; y ofensivas, donde los ataques preventivos o por sorpresa permitieran a las FDI mantener la iniciativa en todos los frentes y garantizar que sus fuerzas - con enorme movilidad táctica pero con una potencia de fuego limitada - se enfrentaran con garantías de éxito a todos sus enemigos. Finalmente, estas guerras se tenían que librar en territorio enemigo para mantener intactos los centros de población israelíes mientras las FDI aprovechaban su mayor movilidad táctica para ganar terreno enemigo y conducir sus operaciones ofensivas con mayores garantías de éxito.

Los ejemplos paradigmáticos de este tipo de operaciones son la guerra de los Seis Días, la campaña del Sinaí o la invasión del Líbano. Por contra, la guerra del Yom Kippur (y, en cierta manera, la campaña de verano de 2006 contra Hezbollah) representa el caso

territorio israelí. Para analizar con más detalladamente la doctrina nuclear hebrea es muy recomendable la lectura de Hersh, Seymour (1991): The Samson Option, Israel's Nuclear Arsenal, Nueva York, Foreign Policy Press o Cohen, Avner (1998): Israel and the bomb, Nueva York, Columbia University Press.

${ }^{18}$ Inbar, Ephraim y Sandler, Shmuel: "Israel's Deterrence Strategy Revisited", Security Studies Vol. 14 No 3 (Invierno 1993), pp. 13-28; en: www.informaworld.com/smpp/ftinterface $\sim$ content=a 789438841 fulltext=713240930 frm=content; o Dayan, Uzi: "Israel's Deterrence after the Second Lebanon War", Israel Security, Vol. 6 No 19 (Febrero 2007), s.n., en: www.jcpa.org/JCPA/Templates/ShowPage.asp ?DBID=1\&TMID=111\&LNGID=1\&FID

19 Katz, Yakov: “Analysis: Israel's deterrence needs a boost”, Jerusalem Post (8 de Abril de 2010), en: www.jpost.com/Israel/Article.aspx?id=183566

${ }^{20}$ Según Heller, continuity and Change in Israeli Security Policy, op. cit., pp. 13-19, la política nacional de seguridad israelí sirve al objetivo político de la paz sólo si existe una superioridad militar real capaz de disuadir a los adversarios árabes de iniciar una guerra, obligándoles a renunciar a ella como opción política. Sin embargo, también afirma que Israel nunca tendrá ni los recursos ni la libertad de acción para conseguir una victoria estratégica - la capacidad de imponer su paz en los términos de un adversario vencido - porque los objetivos políticos de Israel no pueden ser trasladados al campo militar. 
contrario y el escenario más temido por las FDI: a causa de un ataque sorpresa por parte de fuerzas sirias por el norte y egipcias por el sudoeste, el ejército israelí se vio obligado a combatir dentro de su territorio (cuyo grueso había sido obtenido en 1967 y que le garantizaba profundidad estratégica) contra fuerzas más numerosas que disfrutaban de iniciativa táctica y que atacaban desde dos frentes distintos. Después de este relativo desastre - que pudo evitarse por la profundidad estratégica que brindaba el territorio conquistado durante la Guerra de los Seis Días, la plena movilización de los reservistas y la inestimable ayuda norteamericana - se inició una reestructuración de los ejércitos y la armada con objeto de equilibrar la estructura de fuerzas e incrementar el tamaño de las FDI para conseguir una superioridad aplastante en cualquier guerra convencional. Sin embargo, a nivel operacional mantuvieron la doctrina vigente de combatir manteniendo la iniciativa en guerras cortas y ofensivas.

En conclusión, es posible afirmar que, vista la experiencia histórica y observando las tendencias de los ejércitos modernos, en el campo convencional las FDI combinarán la potencia de fuego, la precisión, el conocimiento del entorno operativo y la movilidad para conducir acciones conjuntas e integradas encaminadas a la consecución de sus objetivos militares - la protección de la población civil y la destrucción de las fuerzas enemigas - en el menor tiempo y con el menor número de bajas posible.

\subsection{El énfasis israelí por la calidad}

La dicotomía entre calidad y cantidad es una cuestión muy recurrente a lo largo de la historia militar, dicotomía que ha resultado paradigmática en el caso israelí. De este modo, durante la Guerra de la Independencia (1948), el embrión de las FDI consideró que la única opción para superar la enorme desigualdad demográfica entre judíos y árabes era crear un ejército intensivo en soldados y en armamento capaz de enfrentarse con garantías de éxito a las fuerzas militares árabes, extensivas en soldados y material ${ }^{21}$. No obstante, la experiencia empírica contrasta con este modelo teórico ya que los árabes no siempre han gozado de superioridad cuantitativa ni los israelíes han poseído mejor armamento que los primeros. El único factor que se ha mantenido constante ha sido la calidad de las tropas israelíes que, mejor educadas, adiestradas y entrenadas que las árabes, les ha permitido maximizar sus capacidades y minimizar la desproporción numérica.

La superioridad israelí en el campo armamentístico es un fenómeno relativamente reciente. Antes de la Guerra de los Seis Días las FDI disponían de armamento de menor calidad que el árabe, puesto que mientras éstos últimos recibían equipos soviéticos nuevos como Siria y Egipto - o poseían armamento británico de gran calidad como Jordania, Israel disponía de anticuados sistemas obtenidos de los excedentes de la Segunda Guerra Mundial. Después de la guerra de 1967, las FDI alcanzaron la superioridad tecnológica en el aire cuando Estados Unidos proporcionó a la fuerza aérea aviones de combate de última generación capaces de transportar armamento nuclear, y la armada incorporó buques de fabricación local con tecnologías indígenas. Por otra parte, el ejército de tierra - el más numeroso y la punta de lanza de todas las operaciones militares que ha lanzado Israel - logró una cierta paridad respecto a sus adversarios árabes ${ }^{22}$. No obstante, esta situación se decantó

\footnotetext{
${ }^{21}$ Téngase en cuenta que la aplicación de Israel de la fuerza ofensiva no buscaba la destrucción física de los ejércitos enemigos, sino disminuir su capacidad para sostener sus operaciones antes que una potencia extranjera impusiera un alto el fuego.

${ }^{22}$ Aunque es difícil resumir la relación entre los sistemas de armas empleados por ambos contendientes, en términos generales se podría afirmar que en equipos individuales y carros de combate existía una cierta paridad, la artillería israelí era inferior pero su capacidad de mando, control y comunicaciones era superior a la de sus adversarios.
} 
por completo después de 1973, cuando el país comenzó a producir en serie sus propios sistemas de armas con el fin de lograr la independencia estratégica en el campo de la guerra terrestre.

En último lugar, la preferencia israelí por un ejército cualitativamente intensivo cambió después de la guerra de Yom Kippur, ya que el desastre moral vivido por el país convenció a las FDI que solamente con calidad no se podía hacer frente a ejércitos más numerosos que operaran con iniciativa estratégica. En consecuencia, Tel Aviv decidió aumentar el tamaño de sus fuerzas armadas y equiparse con sistemas de última tecnología, creando con ello una brecha militar respecto a sus adversarios que no ha parado de aumentar, y que ha convertido a las FDI en el ejército más poderoso de la región. Además, no debe olvidarse que Israel también posee el Arma Absoluta, pues es el único país de Oriente Medio en poseer ingenios nucleares y vectores capaces de batir cualquier objetivo enemigo se encuentre donde se encuentre.

\section{El cambiante entorno regional}

Aunque el riesgo de guerra convencional se alzaba como la mayor amenaza que se cernía sobre la integridad de Israel hasta 1973, los cambios políticos en la región derivados de la guerra de Yom Kippur (el proceso de paz entre Israel y Egipto, el intento fallido de Siria por conseguir la paridad militar con Israel y la determinación iraquí por convertirse en una potencia regional); la creciente brecha militar entre Israel y sus adversarios o la nueva estrategia adoptada por la Organización para la Liberación de Palestina (OLP), provocaron el surgimiento de nuevas amenazas de tipo irregular ${ }^{23}$ y la proliferación de armamento de destrucción masiva. En consecuencia, Israel se vio obligado a trazar posibles respuestas como fortalecer la disuasión o desarrollar medidas de defensa activas dentro y fuera de las fronteras del país.

Pero no fue hasta la década de los ochenta cuando Oriente Medio vio proliferar los misiles balísticos y los agentes químicos o biológicos, una peligrosa combinación que convertía en vulnerables todos y cada uno de los objetivos estratégicos de la región ${ }^{24}$. Este incremento en el número de países con armamento no-convencional (actualmente químico y biológico, y en un futuro nuclear) y con vectores capaces de proyectarla más allá de sus fronteras podrá determinar el surgimiento de unas relaciones regionales basadas en la disuasión multipolar ${ }^{25}$, una situación a la que Israel se está preparando de dos maneras. En primer lugar, reforzando su capacidad de disuasión y respuesta al incrementar su flota submarina, proyectada para garantizar su capacidad de represalia nuclear, y afirmar taxativamente que responderá con ingenios nucleares en caso de un ataque perpetrado por un

\footnotetext{
${ }^{23}$ Aunque a fecha de hoy son Hamas y Hezbollah las organizaciones que emplean medios y tácticas irregulares e híbridas para lograr sus objetivos políticos, inicialmente fue la OLP el actor que utilizó estos métodos para erosionar la sociedad israelí, situar el problema palestino en la vanguardia de la agenda israelí e internacional y forzar la retirada hebrea de los territorios ocupados. Un interesante análisis de la evolución de las distintas estrategias - tanto pacíficas como violentas - empleadas por los palestinos a lo largo de las dos Intifadas puede hallarse en Heller, Mark: "The Arab-Israeli Arena”, en Kam, Ephraim y Shapir, Ytzak (eds.) (2003): The Middle East Strategic Balance 2002-2003, Tel Aviv, Jefe Center for Strategic Studies.

${ }^{24}$ Los misiles balísticos de mayor alcance los posee Israel - hasta $4500 \mathrm{Km}$. - aunque Irán dispone de misiles de medio alcance Shehab III con $1300 \mathrm{Km}$. de autonomía y está desarrollando con tecnología norcoreana vectores con alcances de $4500 \mathrm{Km}$. Por su parte, Siria posee misiles con alcances comprendidos entre $300 \mathrm{y}$ $500 \mathrm{Km}$. e Iraq poseía misiles SCUD (de 300 a $500 \mathrm{Km}$.), Al-Hussein $(650 \mathrm{Km}$.) y Al-Abbas (950 Km.).

${ }^{25}$ Heller, Continuity and Change in Israeli Security Policy, op. cit., pp. 32-34
} 
Estado u actor no-estatal con armas de destrucción masiva...una declaración que todavía hoy esconde una postura nuclear ambigua ${ }^{26}$. En segundo lugar, Israel también parece estar adoptando una estrategia de seguridad más defensiva mediante la construcción de un complejo y costoso escudo antimisiles que permita aislar el país frente a cualquier amenaza procedente de Irán y proteja a la población hebrea de los cohetes lanzados desde el Líbano, Cisjordania o $\mathrm{Gaza}^{27}$. No obstante, cabe recordar que ningún sistema defensivo puede proteger completamente a Israel, de modo que la única opción viable para garantizar la seguridad de este pequeño país mediterráneo es mantener, en la medida de lo posible, las relaciones con sus vecinos y establecer contactos con sus potenciales adversarios a la vez que moderniza e incrementa sus capacidades de disuasión y respuesta.

\section{La respuesta israelí al nuevo entorno regional}

Hace poco más de una década, el ortodoxo Gerald Steinberg afirmó que el poder de Israel en Oriente Medio estaba disminuyendo a causa del impacto de un proceso de paz incierto, el aumento de la capacidad militar iraní, la proliferación de armamento de destrucción masiva y el gradual retorno a las políticas de confrontación entre algunos países de la región ${ }^{28}$. Aunque a día de hoy - con Irán en plena proliferación, Líbano en descomposición, Siria técnicamente fallido, Hezbollah reforzado y Turquía cada vez más islamizada - la situación no es en absoluto esperanzadora $^{29}$, Israel también mantiene fluidas relaciones con su vecino jordano a la vez que continúa, pese al distanciamiento que está tomando la Administración Obama, como aliado preferente de Estados Unidos en el gran Oriente Medio ${ }^{30}$.

Y es que a pesar de que hasta fechas recientes Israel y Turquía disfrutaban de una firme alianza que les proporcionaba multiplicadores estratégicos (ambos mantenían lazos con Estados Unidos, compartían una visión común frente a Siria y sobre la proliferación de armamento de destrucción masiva en la región); son varios los indicios ${ }^{31}$ que sugieren que Ankara no sólo ha dado por terminada esta entente cordial con Tel Aviv sino que está

${ }^{26}$ Freilich, Chuck (2010): "The Armageddon scenario: Israel and the treat of nuclear terrorism", Mideast Security and Policy Studies $\mathrm{N}^{\mathrm{o}}$ 84, Ramat Gan: Bar Illan University, en: www.biu.ac.il /SOC/besa/MSPS84.pdf

${ }^{27}$ Este escudo antimisiles ha recibido el nombre de Homa - muro en hebreo - e incluye sistemas BMD (Defensa de mísiles balísticos) para interceptar objetos en las últimas fases de vuelo (el misil Arrow, el Patriot PAC-2 y el control de tiro asociado Green Pines, en fase de despliegue), sistemas BPI (Interceptación primaria de misiles balísticos) con el objetivo de destruir vectores balísticos y de crucero en las primeras etapas de vuelo, mientras se mueven lentamente y representan blancos fáciles; y defensa de punto - una de las grandes lecciones aprendidas de la guerra de verano de 2006 - frente a los cohetes Katyusha, Qassam, Fajr-3, Fajr-5 o Zelzal-2 lanzados desde el Líbano, Gaza o Cisjordania. Hugues, Robin: “Tier Presure”, Jane's Defence Weekly (18 de Julio de 2007).

${ }^{28}$ Steinberg, Gerald (1999): Re-examining Israel's security doctrine, Londres, Royal United Services Institute for Defence Analysis, en: www. faculty.biu.ac.il/ steing/arms/doctrine.htm

${ }^{29}$ Beres, Louis: "Israel's Uncertain Strategic Future”, Parameters Vol. XXXVII No 1 (Primavera 2007), pp. 3754, en: www.carlisle.army.mil/usawc/parameters/Articles/07spring/beres.pdf

${ }^{30}$ Para que Israel continúe gozando de la hegemonía en Oriente Medio, es imprescindible que mantenga el diálogo estratégico con Estados Unidos. Esta relación, basada en intereses geoestratégicos comunes, se vio reforzada por la política exterior norteamericana en la región después del 11-S pero recientemente se ha visto templada por el Presidente Barack Obama.

${ }^{31}$ En este sentido, puede que el más conocido por el público español haya sido el desafortunado incidente del buque $M V$ Mavi Marmara en el que se vieron involucrados ciudadanos de nuestro país. Caro, Laura: "Israel ataca la flota con ayuda para Gaza y causa entre 9 y 19 muertos", $A B C$ (30 de Mayo de 2010), en: www.abc.es/20100531/internacional-/israel-barco-gaza-201005310606.html 
empezando a centrar su mirada en Oriente ${ }^{32}$. Al mismo tiempo, Israel mantiene fluidas relaciones con Jordania que, articuladas en torno a la cuestión Palestina y a necesidades de seguridad comunes, aíslan a Siria de las relaciones regionales. Atendiendo a estos avances diplomáticos, es posible afirmar que si en un futuro se resolviera satisfactoriamente la cuestión Palestina y la situación sirio-libanesa, ambos países podrían establecer el núcleo de una estructura de seguridad regional frente a la amenaza persa. En caso de producirse, ésta podría contar con la participación de los países del Golfo Pérsico siempre y cuando consigan mantener un statu quo cada vez más precario, además de la presencia de Turquía si ésta optara por reevaluar sus intereses estratégicos, y la eventual participación de Egipto dependiendo de su evolución política interna tras la era Mubarak.

A pesar de todo ello, parece evidente que cualquier hipotético avance diplomático entre Israel y sus vecinos estará supeditado a la suspensión - o restricción en el número, alcance y violencia - de las acciones militares preventivas, un recurso todavía posible, plausible y necesario a ojos de la comunidad de defensa hebrea pero cuyos costes diplomáticos, políticos, sociales y mediáticos son cada vez más elevados, tal y como demuestran las opiniones de la comunidad internacional.

\section{Israel ante el cambio de paradigma estratégico}

Tal y como se ha señalado en las páginas anteriores, el paradigma estratégico israelí se ha fundamentado en unos grandes principios que, trazados durante la creación del país y revisados después del desastre de 1973, han permanecido casi inalterables hasta la pasada década, cuando una Revolución en la Seguridad Israelí obligó a reformular varios de los fundamentos del modelo vigente hasta la fecha. Hoy en día, otro cambio mucho más profundo parece estarse gestando en la arquitectura de seguridad y defensa del país con objeto de resolver los problemas identificados en la campaña de verano de 2006 y adaptar este modelo a la cambiante coyuntura regional.

A grandes rasgos, las importantes transformaciones que han sufrido las FDI - tanto en lo que respecta a su estructura de fuerzas y catálogo de capacidades militares como a sus relaciones con la sociedad civil y el poder político - responden a lo que Cohen, Eisenstadt y Bacevich definen como una Revolución en la Seguridad Israelí que está erosionando los fundamentos políticos, sociales y militares del modelo de seguridad hebreo ${ }^{33}$. Este conjunto de cambios, fácilmente equiparables a lo que en el resto del globo se denominó Revolución en los Asuntos Militares - un profundo cambio en la forma de combatir vinculada con el advenimiento de la Era de la Información capaz de incrementar el potencial militar de los países avanzados y lograr victorias rápidas, decisivas y sin apenas daños colaterales o bajas propias - pretende preservar la supremacía militar israelí mediante una profunda transformación de sus fuerzas armadas y de la gestión de su política de defensa ${ }^{34}$.

\footnotetext{
${ }^{32}$ Altunisik, Meliha B.: “Turkey’s Changing Middle East Policy”, UNISCI Discussion Papers N 23 (Mayo 2010), pp. 149-162, en: www.ucm.es/info/unisci/revistas/UNISCI\%20DP\%2023\%20-\%20BENLI.pdf; o Inbar, Efraim: "Turkey says good bye to Israel and the West", Perspectives Papers on Current Affairs No 108 (Junio 2010), en: www.biu.ac.il/SOC/besa/docs/perspectives108.pdf

33 Cohen, Eliot; Eisenstadt, Michael y Bacevich, Andrew (1998): Knives, Tanks, and Missiles: Israel's Security Revolution, Washington DC, Washington Institute for Near East Policy

${ }^{34}$ Colom, Guillem (2008): Entre Ares y Atenea: el debate sobre la Revolución en los Asuntos Militares, Madrid, Instituto Universitario General Gutiérrez Mellado, en: www.iugm.es/uploads/tx_iugm /Libro_Entre_ares.pdf
} 
En este sentido, las modernas tecnologías vinculadas con esta revolución - avanzados sistemas de mando, control y comunicaciones, armamento de precisión e inteligente o sofisticadas plataformas terrestres, navales y aéreas - están proporcionando a las FDI la capacidad para batir prácticamente cualquier objetivo convencional enemigo con una precisión y efectividad sin precedentes. Ello ha resuelto una de las grandes demandas de la sociedad israelí (reducir el nivel de bajas propias y garantizar la protección de la fuerza) y ha determinado importantes cambios en el despliegue militar (utilización extensiva de sistemas tecnológicamente avanzados en operaciones diarias de seguridad y antiterrorismo), en su composición interna (limitado empleo de reservistas y abandono del servicio militar universal) ${ }^{35}$ y en la organización del ejército (reducción de los objetivos de fuerza, proporción entre los catálogos de capacidades y aumento de la potencia de fuego de todas las unidades).

Sin embargo, al aceptar todos los principios de esta Revolución en los Asuntos Militares, Israel ha americanizado en gran medida la doctrina, organización, táctica y tecnología de las $\mathrm{FDI}^{36}$, una realidad que ha generado enormes resistencias entre las filas de un ejército ávido por preservar sus rasgos distintivos y mantener intacta su influencia sociopolítica $^{37}$. Además, esta revolución discurre en paralelo a la fractura que existe entre la esfera civil y militar del país provocada por el advenimiento de una nueva era post-heroica ${ }^{38}$ en la que los valores post-modernos y post-materialistas se han adueñado de la sociedad hebrea $^{39}$. Y es que en esta nueva etapa histórica Israel no sólo se está redefiniendo la defensa nacional y el papel de sus fuerzas armadas como fundamento de la sociedad hebrea, pues hoy en día la percepción que se tiene de ellas es negativa debido a la necesidad de realizar labores calificadas como sucias (antiterrorismo, asesinatos selectivos u ocupación) que si bien garantizan la seguridad diaria del país, también alteran el clásico - y romántico en numerosos casos - planteamiento de las FDI como una fuerza puramente convencional preparada para la defensa del territorio patrio frente a cualquier invasión árabe y degradan la capacidad operativa del ejército al distraer efectivos para llevar a cabo labores de guerra irregular.

Los enormes cambios provocados por la Revolución en la Seguridad Israelí y la entrada del país en la era post-heroica se pusieron de manifiesto en la guerra de verano de 2006: la Operación Recompensa Justa, que enfrentó a Israel contra Hezbollah - un actor noestatal que se había estado preparando durante años para repeler una incursión terrestre israelí en el sur del Líbano - no sólo reveló el cambiante rostro de la guerra, el creciente divorcio de

${ }^{35}$ En efecto, el abandono del sistema militar universal coincidirá con la adopción de un sistema híbrido que mantendrá el principio de universalidad para hombres y mujeres aunque estableciendo diferentes métodos de alistamiento (reclutas con un servicio militar básico seguido por tareas en la reserva al estilo suizo, voluntarios con un período mas largo de servicio activo o profesionales de carrera). Esta fórmula permitirá contener la creciente tensión entre la sociedad israelí y sus fuerzas armadas y maximizar la nueva estructura demográfica del país.

${ }^{36}$ Gal, Reuven y Cohen, Stuart: "Israel: Still Waiting in the Wings", en Moskos, Charles; Williams, James y Segal, Don (2000): The Post-Modern Military: Armed Forces after the Cold War, Nueva York, Oxford University Press.

${ }^{37}$ Maoz, Zeeb (2004): The Revolution in Military Affairs and the Middle East: if this is a Revolution, then we are the Counterrevolutionists, National Intelligence Council Discussion Paper, Washington DC: U.S. Government Printing Office, en: www.dni.gov/nic/PDF_GIF...05.../military_affairs.pdf

${ }^{38}$ Luttwak, Edward, N.: “A Post-Heroic Military Policy”, Foreign Affairs, Vol. 75 № 4 (Julio-Agosto 1996), pp. 33-44

${ }^{39}$ En efecto, y es que a diferencia del pasado, donde la sociedad israelí había integrado el ethos guerrero, hoy en día esta sociedad avanzada presenta importantes debilidades como la volubilidad de su opinión pública doméstica, el pánico a las bajas propias y el temor a los daños colaterales, el sometimiento a unos usos y costumbres de la guerra restrictivos y anacrónicos, una relativa ansiedad por los costes políticos y efectos electorales de las operaciones, la exigencia de limitar su alcance, impacto y duración, la necesidad de emplear la fuerza de manera limitada y restrictiva o la incapacidad de implementar estrategias integrales a largo plazo. 
la sociedad israelí con sus fuerzas armadas y la erosión del tradicional modelo estratégico hebreo; sino que también expuso las carencias de una fuerza equipada, adoctrinada, entrenada y preparada para el combate convencional cuando se vio forzada a luchar en una guerra urbana contra un adversario híbrido (que integra la lucha irregular o contraria a los usos y costumbres de la guerra con acciones convencionales de cierta complejidad ${ }^{40}$ ) y el grave error que ha supuesto la americanización de las FDI. Todo ello está obligando a revisar nuevamente la estructura de fuerzas, el catálogo de capacidades y los niveles de ambición para adaptarlos al nuevo entorno estratégico; y reestablecer las relaciones entre los líderes políticos y los mandos militares para que no vuelva a repetirse una situación como la de $2006^{41}$.

La resolución a estos problemas y la definición de un nuevo paradigma estratégico adaptado a la realidad política, social, militar e internacional presente y futura marcará la gestión de la seguridad y la defensa israelíes durante los próximos años.

\section{Conclusiones}

Con más de medio siglo de existencia en su haber, el Estado de Israel todavía basa su modelo de seguridad nacional en unos principios que trazó durante su fundación, desarrolló a lo largo de sus primeros años de vida y que revisó después de la debacle de 1973. Aunque este modelo indefinido, pragmático, fundamentado en la autonomía militar y en el arquetipo de ciudadanosoldado le ha permitido cosechar importantes éxitos en las guerras pasadas y adaptarse con inusitada flexibilidad a todos los cambios que se han producido en Oriente Medio, transformaciones internas como la Revolución en la Seguridad Israeli o la entrada del país en una era post-heroica, y externas como la proliferación de armamento de destrucción masiva, un entorno regional en constante evolución y la creciente amenaza irregular e híbrida, están obligando su redefinición.

La guerra de verano de 2006 reveló el alcance de este conjunto de cambios que se habían estado gestando durante las décadas anteriores. Aunque el empate técnico logrado por las milicias chiítas de Hezbollah frente al todopoderoso ejército israelí se debe a la conjunción de varios factores (una incorrecta planificación militar, un lento proceso de toma de decisiones, graves errores de inteligencia o una deficiente gestión de la información pública en el caso israelí; y el empleo de métodos y medios híbridos con enorme acierto, la defensa a distintos niveles y en profundidad del territorio libanés, una eficaz labor de inteligencia y una excelente explotación mediática de todas las victorias tácticas en el caso de Hezbollah), casi todos ellos estaban relacionadas con la inadecuación del modelo de seguridad hebreo a la realidad política, estratégica, táctica y doméstica del primer cuarto del siglo XXI.

Aunque constituida para depurar la actuación de los mandos políticos y militares durante la guerra de verano de 2006, la Comisión Winograd - heredera de la Comisión Agranat que evaluó la guerra del Yom Kippur y sus conclusiones sirvieron para adaptar la

\footnotetext{
${ }^{40}$ Hoffman, Frank G. (2007): Conflict in the $21^{\text {st }}$ Century: the Rise of Hybrid Wars, Arlington, Potomac Institute for Policy Studies, en: http:/www.potomacinstitute.org/images/stories/publications/potomac hybridwar_0108.pdf

${ }^{41}$ Una visión general de la campaña puede hallarse en: Harel, Amos y Isacharoff, Avi (2008): 34 Days: Israel, Hezbollah and the War in Lebanon, Nueva York: Palgrave MacMillan; mientras que para un análisis centrado en los asuntos militares es muy recommendable la lectura de Matthews, Matt (2008): We Were Caught Unprepared: the 2006 Hezbollah-Israeli War, Fort Leavenworth, U.S. Combat Studies Institute, 2008, en: www.handle.dtic.mil/100.2/ADA477851
} 
seguridad y la defensa del país al mundo de los años setenta - ha permitido identificar los errores que condujeron a la debacle israelí y ha propuesto una serie de medidas correctivas para evitar que pueda producirse en el futuro otra situación similar.

Al identificar los errores y proponer posibles soluciones para atajarlos, la Comisión Winograd pretende acomodar el modelo de seguridad y defensa nacional hebreo a los retos estratégicos actuales y futuros a la vez que refuerza la hegemonía militar del país en la región ${ }^{42}$. Para ello, el informe no sólo propone la completa revisión de la arquitectura de seguridad nacional israelí (reformulando el proceso de toma de decisiones, integrando los procesos de planeamiento civil y militar, reorganizando la inteligencia, consolidando el control político sobre la autonomía militar de las FDI, estableciendo una estrategia integral que equilibre las necesidades militares con las limitaciones políticas, creando una nueva estrategia disuasoria o potenciando el Consejo de Seguridad Nacional) y una profunda reestructuración de sus Fuerzas Armadas (integrando las lecciones aprendidas de las campañas recientes y desarrollando una estructura de fuerzas y un catálogo de capacidades equilibrados que le permitan luchar en toda la gama de las operaciones y triunfar sobre cualquier adversario convencional, irregular o híbrido); sino también promover profundos cambios en la diplomacia pública del país, en la estrategia de comunicación doméstica e internacional, en las relaciones civiles-militares, en la percepción de la amenaza o en la cultura de defensa hebrea.

Aunque es muy probable que este conjunto de cambios acaben con el tradicional paradigma estratégico que tantos éxitos ha brindado a Israel a lo largo de su corta historia y termine con algunos de los rasgos definidores de su modelo militar; también garantizará la adaptación de este pequeño país mediterráneo al heterogéneo y cambiante entorno estratégico de inicios del tercer milenio.

\footnotetext{
${ }^{42}$ Spanó, Vincenzo: "El informe de la Comisión Winograd y sus consecuencias", VV.AA. (2009): Nuevas Guerras y Polemología, Madrid: Centro Superior de Estudios de la Defensa Nacional, pp. 185-223, en: www.ceseden.es/centro_documentacion/monografias/111.pdf
} 